\title{
Pulmonary function and sputum characteristics predict computed tomography phenotype and severity of COPD
}

\author{
Gianna Camiciottoli, Francesca Bigazzi, Matteo Paoletti, Lucia Cestelli, \\ Federico Lavorini and Massimo Pistolesi
}

Affiliations: Section of Respiratory Medicine, Dept of Experimental and Clinical Medicine, University of Florence, Florence, Italy.

Correspondence: M. Pistolesi, Dept of Experimental and Clinical Medicine, Section of Respiratory Medicine, University of Florence, Largo Brambilla 3, 50134 Firenze, Italy. E-mail: massimo.pistolesiQunifi.it

ABSTRACT Airway obstruction and parenchymal destruction underlie phenotype and severity in chronic obstructive pulmonary disease (COPD). We aimed to predict, by clinical and pulmonary function data, the predominant type and severity of pathological changes quantitatively assessed by computed tomography (CT).

Airway wall thickness (AWT-Pi10) and percentage of lung area with X-ray attenuation values $<-950 \mathrm{HU}$ (\%LAA-950) were measured in 100 (learning set) out of 473 COPD outpatients undergoing clinical and functional evaluation. Original CT measurements were translated by principal component analysis onto a plane with the novel coordinates CT1 and CT2, depending on the difference (prevalent mechanism of airflow limitation) and on the sum (severity) of AWT-Pi10 and \%LAA-950, respectively. CT1 and CT2, estimated in the learning set by cross-validated models of clinical and functional variables, were used to classify 373 patients in the testing set.

A model based on diffusing capacity of the lung for carbon monoxide, total lung capacity and purulent sputum predicted CT1 $(\mathrm{r}=0.64 ; \mathrm{p}<0.01)$. A model based on forced expiratory volume in $1 \mathrm{~s} / \mathrm{vital}$ capacity, functional residual capacity and purulent sputum predicted CT2 $(r=0.77 ; \mathrm{p}<0.01)$. Classification of patients in the testing set obtained by model-predicted CT1 and CT2 reflected, according to correlations with clinical and functional variables, both COPD phenotype and severity.

Multivariate models based on pulmonary function variables and sputum purulence classify patients according to overall severity and predominant phenotype of COPD as assessed quantitatively by CT.

@ERSpublications

Pulmonary function and sputum purulence models classify COPD patients by severity and phenotype as quantified by CT http://ow.ly/kyP6d

This article has supplementary material available from www.erj.ersjournals.com

Received: Aug 242012 | Accepted after revision: Nov 292012 | First published online: Dec 202012

Support statement: This work was funded by a grant from the Center for Diseases Control (CCM) of the Ministry of Health, Italy.

Conflict of interest: Disclosures can be found alongside the online version of this article at www.erj.ersjounals.com

Copyright @ERS 2013 


\section{Introduction}

Chronic obstructive pulmonary disease (COPD) is diagnosed in the presence of a ratio of forced expiratory volume in $1 \mathrm{~s}$ (FEV1) to forced vital capacity (FVC) $<70 \%$, and assessed for severity according to the level of expiratory airflow limitation as measured by FEV1 [1]. Clinical conditions whose pathogenetic mechanisms and degrees of severity could substantially differ are unified under the term COPD by the spirometric detection of airflow limitation. FEV1 alone and its ratio to FVC can be considered reliable screening tools because they reflect both airway obstruction and emphysema. However, spirometry does not provide by itself an overall evaluation of the complexity and the severity of COPD [2-6]. The concept that expiratory airflow limitation, the hallmark of COPD, could be the common result of different lung pathologic changes, such as the obstruction of the conductive airways, the destruction of the lung parenchyma or various combinations of the two, has evolved from the seminal observations of the middle decades of the twentieth century [7] to the current explosion of interest in identifying the clinical phenotypes of COPD [8]. Recently, respiratory symptoms and prevalence of exacerbations have been introduced as new dimensions for the definition and grading of COPD in individuals $[9,10]$.

Quantitative computed tomography (CT) studies of COPD opened a new era for the understanding of the mechanisms underlying airflow limitation. CT can provide in vivo information to assess the severity of the disease and to differentiate patients with predominantly conductive airway obstruction from those with predominantly emphysematous destruction [11-17]. Recent software developments for CT data analysis have increased the reliability of the measurements of the pathological changes occurring in COPD lungs. In general, there is evidence that quantitative CT data identify the predominant phenotype [18-22] and assess the overall severity [23-27] of COPD with considerable accuracy. However, considering the high prevalence of COPD in the general population, the cost of the examination, and the risks associated with the radiation exposure necessary to obtain a CT scan [28], it is evident that quantitative CT scan data cannot, at present, be obtained in all patients affected by COPD. Further technological improvements of CT may possibly increase its use in the future in patients, such those with COPD, in whom the age-related risk of radiation exposure could be substantially low. It remains to be ascertained whether and to what extent evaluation by quantitative CT can be related to the conventional characterisation of COPD by accurate clinical history and lung function evaluation entailing, in addition to spirometry, static lung volumes and diffusing capacity.

The aim of this study was to establish a link between quantitative CT data on lung density and airway wall thickening [22, 29] and clinical and whole pulmonary function evaluation in a series of patients affected by COPD. In particular, by using a statistical approach allowing the classification of patients by large sets of variables, avoiding a priori expectations about disease characteristics, we wanted to ascertain whether the overall severity and the predominant type of the lung pathologic changes quantitatively assessed by CT could be predicted by clinical and pulmonary function data.

\section{Methods \\ Patients and study design}

This single-centre cross-sectional study was approved by the Ethics Committee of the Florence University Hospital (Florence, Italy). All participants provided written informed consent. From September 2010 to September 2011 we recruited 473 outpatients (98 females) with COPD through the outpatient clinic. Patients recruited are some of those participating in the Clinical Identification of the Phenotypes of COPD study. Diagnosis of COPD was based on Global Initiative for Chronic Obstructive Lung Disease (GOLD) guidelines [1]. We included patients aged 40-85 years with COPD in GOLD stages I-IV and a smoking history of $\geqslant 10$ pack-years who showed nonreversible post-bronchodilator airflow obstruction. We excluded subjects within 1 month of an exacerbation or who had clinical conditions that could interfere with the assessment of pulmonary function or chest CT quantitative parameters, including asthma, diffuse bronchiectasis, cystic fibrosis, interstitial lung disease, acute heart failure, chemo-/radiation therapy, lung cancer, lung surgery, known or suspected pregnancy and metal objects in the chest.

All patients underwent full clinical and functional lung evaluation. The study was designed to obtain in a group of patients (the learning set) quantitative CT parameters evaluating lung density and airway wall thickness. By means of a data-driven statistical procedure, we derived from the original CT parameters two CT indexes that aimed to reflect both the underlying mechanisms of airflow limitation and the overall severity of the disease. Considering clinical and functional data as independent variables, we developed two multivariate models to predict the two CT indexes in the learning set. Model-predicted CT indexes were then used to classify a second, larger group of patients not undergoing CT scanning (the testing set). Inclusion in the learning or the testing set was not based on predefined selection criteria, but on the patient's willingness to participate in the CT study and on the availability of the CT equipment within $48 \mathrm{~h}$ of the clinical and functional evaluation. 


\section{Clinical and functional evaluation}

Evaluation of patients consisted of a thorough clinical history focused on respiratory symptoms, such as cough frequency and sputum characteristics, and administration of the modified Medical Research Council (mMRC) questionnaire for the assessment of dyspnoea severity. Cough was scored as 0 if absent, 1 if occasional and 2 if chronic. Sputum was scored as 0 if absent/occasional, 1 if chronic nonpurulent and 2 if chronic purulent.

Static and dynamic lung volumes and single breath diffusing capacity of the lung for carbon monoxide (DLCO) were measured by a mass-flow sensor and a multi-gas analyser (V6200 Autobox Body Plethysmograph; Sensor Medics, Yorba Linda, CA, USA) according to American Thoracic Society (ATS)/ European Respiratory Society (ERS) guidelines, and expressed as percentages of the predicted values [30].

\section{CT data acquisition and analysis}

100 (learning set) out of the 473 patients underwent volumetric chest CT at full inspiration. Patients were previously trained to inspire at total lung capacity (TLC) while lying in the CT acquisition bed. CT scans were obtained by the same team of diagnostic radiology personnel using the same CT scanner (Sensation 64; Siemens, Erlangen, Germany) to ensure uniformity of image acquisition. CT scans were acquired at $120 \mathrm{kVp}, 200 \mathrm{mAs}$, standard B31f reconstruction kernel, slice thickness of $0.75 \mathrm{~mm}$ and slice interval of $0.5 \mathrm{~mm}$. CT examinations were analysed using the software Apollo 1.1 (VIDA Diagnostics Inc., Coralville, IA, USA). The extent of parenchymal destruction was assessed by measuring the percentage of lung area with X-ray attenuation values <-950 HU (\%LAA-950). To overcome possible errors deriving from sampling bias and from different sizes of the airways in different individuals, the dedicated software standardises the measure of airway wall thickness for each patient by plotting the square root of the airway wall area against the internal perimeter of each airway cut in cross-section [31]. The resulting regression line is used to predict the square root of the wall area for an arbitrary airway with an internal perimeter of $10 \mathrm{~mm}$ and to calculate its average wall thickness (AWT-Pi10) [20, 29, 31, 32].

\section{Statistical analysis}

Pearson's correlation analysis was used to study the association between quantitative variables; ANOVA was used to analyse the relationships between quantitative and clinical categorical variables. t-test and ANOVA were used to compare the means of quantitative variables among different subsets of patients. Chi-squared test was performed to compare the prevalence of categorical variables. Significance was set at $p<0.05$. Principal component analysis (PCA) [33] was used to derive two novel parameters (CT1 and CT2) from the originally measured CT variables (\%LAA-950 and AWT-Pi10), combining the information contained therein. PCA is a data-driven statistical technique that can be applied to large sets of overlapping variables to translate original data to a new coordinates system. In this instance, with two original variables, PCA computes two novel coordinates (CT1 and CT2). CT1 is proportional to the difference between the original CT variables (\%LAA-950 and AWT-Pi10), hence representing the prevailing mechanism of airflow limitation (parenchymal destruction or conductive airway obstruction) and, consequently, the COPD phenotype. CT2 is proportional to the sum of the two original variables, hence representing COPD severity resulting from both parenchymal destruction and conductive airway obstruction. Further details about PCA and other statistical procedures are provided in the online supplementary material.

Two linear regression models were trained to estimate CT1 (phenotype) and CT2 (severity) of the learning set using functional and clinical data as independent variables. The most significant models with the least number of variables were selected by a forward stepwise process. Parameters were entered or removed from the process according to F-statistics. Tenfold cross-validation was used to study the selected models' Rshrinkage and to get the cross-validated standard errors of prediction (error mean, error mode) [34]. Briefly, the original dataset of 100 patients was randomly partitioned into 10 subsamples. Each one of the subsamples was used as an independent dataset for testing the model and the remaining nine subsamples were used as training data. The cross-validation process was repeated 10 times with each of the subsets used once as validation set. The best predictive models obtained were applied prospectively to predict by clinical and functional data the phenotype (CT1) and the severity (CT2) in the testing set of 373 patients not undergoing CT scanning. Patients included in the testing set were plotted in a bidimensional plane with model-predicted CT1 and CT2 coordinates. Two perpendicular lines drawn through the centre point of the diagram partitioned the testing set into four subsets.

Data analysis and statistics were performed using SPSS/PC WIN 11.5.1 (SPSS, Chicago, IL, USA), Mathcad (version 2001; MathSoft, Cambridge, MA, USA), and C++ programming language. 
TABLE 1 Continuous variables lanthropometric data, smoking, pulmonary function and computed tomography (CT) measurements) of 473 chronic obstructive pulmonary disease patients

\begin{tabular}{|c|c|c|c|}
\hline & Learning set & Testing set & p-value \\
\hline Subjects $n$ & 100 & 373 & \\
\hline Age years & $63 \pm 10$ & $68 \pm 8$ & $<0.01$ \\
\hline BMI & $25.6 \pm 4$ & $26.3 \pm 5$ & NS \\
\hline Smoking pack-years & $50.4 \pm 36$ & $48.5 \pm 30$ & NS \\
\hline FVC $\%$ pred & $84.6 \pm 24$ & $85.4 \pm 19$ & NS \\
\hline FEV $1 \%$ pred & $55.9 \pm 24$ & $60.9 \pm 20$ & $<0.05$ \\
\hline TLC $\%$ pred & $109.8 \pm 17$ & $106 \pm 16$ & $<0.05$ \\
\hline RV \% pred & $148.1 \pm 53$ & $138.7 \pm 40$ & $<0.05$ \\
\hline RV/TLC & $51.2 \pm 11$ & $50.7 \pm 10$ & NS \\
\hline FRC \% pred & $131.7 \pm 34$ & $123 \pm 30$ & $<0.05$ \\
\hline VC \% pred & $92.2 \pm 21$ & $91 \pm 18$ & NS \\
\hline IC \% pred & $90.8 \pm 26$ & $92.8 \pm 22$ & NS \\
\hline
\end{tabular}

Data are presented as mean \pm SD, unless otherwise stated. BMI: body mass index; pack-years: number of packs of cigarettes per day multiplied by the number of smoking years; FVC: forced vital capacity; \% pred: \% predicted; FEV1: forced expiratory volume in 1 s; VC: vital capacity; TLC: total lung capacity; RV: residual volume; FRC: functional residual capacity; IC: inspiratory capacity; DLCO: single breath diffusion capacity of the lung for carbon monoxide; \%LAA-950: relative attenuation area with X-ray attenuation <-950 HU; AWT-Pi10: average wall thickness of an arbitrary airway with an internal perimeter of $10 \mathrm{~mm}$; NS: not significant. ${ }^{\#}$ : reported only for the 100 patients of the learning set who underwent CT scanning.

\section{Results}

Table 1 reports anthropometric data, smoking exposure, pulmonary function and CT data of the 100 patients of the learning set ( 23 of whom were female) and of the 373 patients of the testing set (75 of whom were female). CT data are reported only for patients in the learning set. Noticeably, both in the learning set and the testing set the value of FEV1/FVC was higher than that of FEV1/vital capacity (VC). Table 2 reports the data on mMRC dyspnoea score and characteristics of cough and sputum. Patients of the learning set were significantly younger, more obstructed and hyperinflated. Sputum purulence prevailed in patients of the testing set. Cardiovascular disease and diabetes prevailed in patients of the testing set, while other

TABLE 2 Categorical variables (modified Medical Research Council (mMRC) dyspnoea score, cough, sputum) of 473 chronic obstructive pulmonary disease patients

\begin{tabular}{|c|c|c|c|}
\hline & Learning set & Testing set & p-value \\
\hline Subjects n & 100 & 373 & \\
\hline mMRC 1 (slight) & 23 & 18 & NS \\
\hline mMRC 2 (moderate) & 33 & 39 & NS \\
\hline mMRC 3 (severe) & 24 & 28 & NS \\
\hline Cough 1 (occasional) & 36 & 27 & NS \\
\hline Cough 2 (chronic) & 49 & 55 & NS \\
\hline Sputum 0 (absent/occasional) & 39 & 32 & NS \\
\hline Sputum 1 (chronic nonpurulent) & 31 & 20 & $<0.05$ \\
\hline Sputum 2 (chronic purulent) & 30 & 48 & $<0.01$ \\
\hline
\end{tabular}

Data are presented as \%, unless otherwise stated. Dyspnoea on effort has been scored into five categories according to the mMRC dyspnoea scale. None: not troubled by shortness of breath except with strenuous exercise; slight: troubled by shortness of breath when climbing a flight of stairs, hurrying on the level or walking up a slight hill; moderate: walks slower than people of the same age on the level because of shortness of breath; severe: stops for breath after walking $\sim 100$ yards or after a few minutes on the level; very severe: too breathless to leave the house or breathless when dressing or undressing. NS: not significant. 

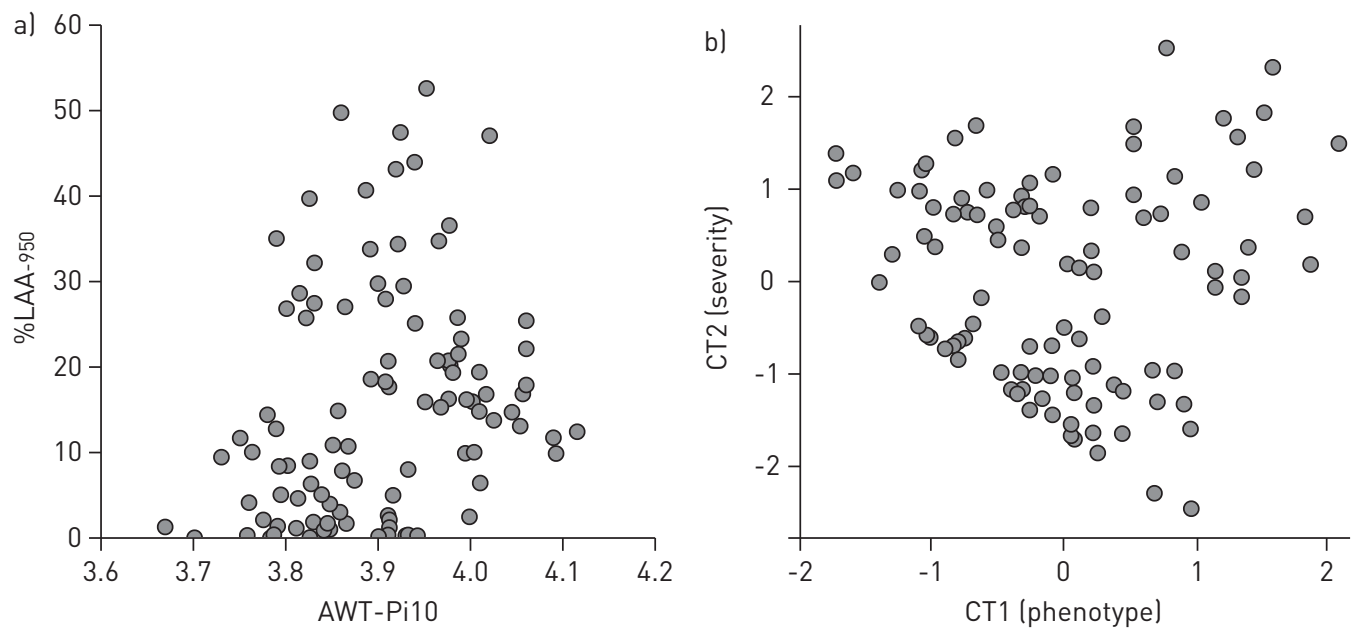

FIGURE 1 a) Relationships between the original computed tomography (CT) measurements (average wall thickness of an arbitrary airway with an internal perimeter of $10 \mathrm{~mm}$ (AWT-Pi10) and percentage of lung area with X-ray attenuation values <-950 HU (\%LAA-950)) in the 100 patients of the learning set; b) results of the application of principal components analysis to the original CT measurements; the information contained in AWT-Pi10 and \%LAA-950 is merged into two components (CT1 and CT2).

common comorbidities of COPD, such as osteoporosis, obesity and depression, had the same prevalence in the two sets of patients.

Figure 1a displays the relationships between the original CT measurements (AWT-Pi10 and \%LAA-950) in the 100 patients of the learning set. The two variables are associated $(r=0.25 ; p<0.01)$, but with great dispersion of the data points. This indicates that in this series of patients conductive airway wall thickening and parenchymal destruction make independent contributions to airflow limitation. Figure $1 \mathrm{~b}$ shows the results of the application of PCA to the original CT measurements; the information contained in AWT-Pi10 and \%LAA-950 is merged in two components (CT1 and CT2). By definition (see the online supplementary material), increasing CT1 values means that parenchymal destruction (\%LLA-950 contribution) is predominant on conductive airway obstruction (AWT-Pi10 contribution), and vice versa. Increasing CT2 values means that the overall severity is increased (combined contributions of \%LLA-950 and AWT-Pi10).

As shown in table 3, there were significant relationships between CT1 and CT2 and lung function tests. CT1 had the highest correlation with DLCO and CT2 with FEV1/VC.

CT1 of the patients of the learning set progressively increased $(p<0.01)$ from a mean value of -0.25 with chronic cough to 0.13 with occasional cough and 0.52 with absent cough. CT1 progressively increased $(\mathrm{p}<0.01)$ from a mean value of -0.45 with chronic purulent sputum to 0.09 with chronic nonpurulent sputum and 0.42 with absent/occasional sputum. These results are compatible with a progressive prevalence of emphysema over conductive airway obstruction with increasing CT1 values. CT2 progressively increased $(\mathrm{p}<0.01)$ from a mean value of -0.73 with mMRC dyspnoea score zero to -0.06 with a score of 2 and 0.64 with a score of 4 . Hence, increased values of CT2 are compatible with increasing severity of COPD resulting from both emphysema and conductive airway obstruction and reflected clinically by worsening of dyspnoea.

TABLE 3 Pearson's correlation coefficients between computed tomography indexes and pulmonary function variables measured in the learning set of 100 patients

\begin{tabular}{|c|c|c|c|c|c|c|c|}
\hline СT1 (phenotype) & $-0.48 * *$ & $-0.19 *$ & $-0.33^{* *}$ & $0.25 *$ & $0.20 *$ & $0.29 * *$ & -0.04 \\
\hline
\end{tabular}

CT1, CT2: numeric indexes resulting from the application of principal component analysis to the relationship of relative lung area with $\mathrm{X}$-ray attenuation $<-950 \mathrm{HU}$ and average wall thickness of an arbitrary airway with an internal perimeter of $10 \mathrm{~mm}$. DLCO: single breath diffusion capacity of the lung for carbon monoxide; FEV1: forced expiratory volume in $1 \mathrm{~s}$; VC: vital capacity; TLC: total lung capacity; RV: residual volume; FRC: functional residual capacity; FVC: forced vital capacity. ${ }^{*}: p<0.05 ;{ }^{* *}: p<0.01$. 
TABLE 4 Predictive models of numeric indexes resulting from the application of principal component analysis to the relationship of relative lung area with X-ray attenuation $<-950 \mathrm{HU}$ and average wall thickness of an arbitrary airway with an internal perimeter of $10 \mathrm{~mm}$ (CT1 and CT2) by multivariate regression analysis of pulmonary function and clinical variables of the learning set of 100 patients

\begin{tabular}{|c|c|c|c|c|c|}
\hline \multirow[t]{2}{*}{ Estimates } & \multirow[t]{2}{*}{ Predictors } & \multirow[t]{2}{*}{ Coefficients } & \multirow[t]{2}{*}{$R / R^{2}$} & \multicolumn{2}{|c|}{ Prediction errors } \\
\hline & & & & Mean & Mode \\
\hline
\end{tabular}

DLCO: single breath diffusion capacity of the lung for carbon monoxide; TLC: total lung capacity; FEV1: forced expiratory volume in 1 s; VC: vital capacity; FRC: functional residual capacity.

Table 4 reports the multivariate models to estimate CT1 (phenotype) and CT2 (severity) by clinical and lung function variables together with their respective regression coefficients and cross-validation predicted errors. Cross-validated R-shrinkage was not significant. The variables entered in the multivariate model to predict CT1 were DLCO \%, TLC \% and purulent sputum, while FEV1/VC, functional residual capacity (FRC $\%$ ), and purulent sputum entered in the model to predict CT2. The model coefficient for purulent sputum was negative in the model predicting CT1 and positive in the model predicting CT2.

As shown in table 5, there were significant relationships between the pulmonary function variables measured in the testing set and CT1 and CT2 predicted by multivariate regression models derived from the learning set. The highest correlations were found between predicted CT1 and DLCO \% and between predicted CT2 and FRC \%.

Predicted CT1 increased stepward in the testing set $(\mathrm{p}<0.01)$ from a mean value of -0.26 with chronic cough to 0.23 with absent cough and from a mean value of -0.41 with chronic purulent sputum to 0.30 with absent/occasional sputum. Predicted CT2 increased stepward $(\mathrm{p}<0.01)$ from a mean value of -0.64 with mMRC dyspnoea score zero to a mean value of 0.25 with a score of 4 .

Figure 2 displays the distribution of the 373 patients of the testing set, using as coordinates the values of CT1 and CT2 predicted by linearly combining the coefficients of the models developed from the learning set with the clinical and functional data of each patient of the testing set. Patients' distribution is partitioned into four subsets (A, B, C and D).

Table 6 shows the mean values of the pulmonary function indexes that are significantly different in each subset with respect to all the others. Reported pulmonary function tests indicate that conductive airway obstruction predominates over emphysema in patients whose predicted CT1 coordinate value is below zero (subsets A and C), while emphysema predominates in the patients of the B and D subsets. Accordingly, chronic cough and purulent sputum prevailed significantly $(\mathrm{p}<0.01)$ in subsets $\mathrm{A}$ and $\mathrm{C}$, while the

TABLE 5 Pearson's correlation coefficients between computed tomography (CT) indexes predicted by multivariate regression models from the learning set of 100 patients and pulmonary function variables measured in the testing set of 373 patients

\begin{tabular}{|c|c|c|c|c|c|c|c|}
\hline & DLCo & FEV $1 \%$ & $\mathrm{FEV}_{1} / \mathrm{VC}$ & $\mathrm{TLC} \%$ & RV\% & FRC\% & FVC\% \\
\hline Predicted CT1 (phenotype) & $-0.79 * *$ & $-0.39 *$ & $-0.47 * *$ & $0.43^{*}$ & $0.49 *$ & $0.50 * *$ & -0.10 \\
\hline Predicted CT2 (severity) & $-0.38 * *$ & $-0.62 * *$ & $-0.76 * *$ & $0.62 * *$ & $0.72 * *$ & $0.78^{* *}$ & $-0.20 * *$ \\
\hline
\end{tabular}

DLCO: single breath diffusion capacity of the lung for carbon monoxide; FEV1: forced expiratory volume in 1 s; VC: vital capacity; TLC: total lung capacity; RV: residual volume; FRC: functional residual capacity; FVC: forced vital capacity; CT1 and CT2: CT indexes predicted by multivariate regression models. ${ }^{*}: p<0.05 ;{ }^{* *}: p<0.01$. 


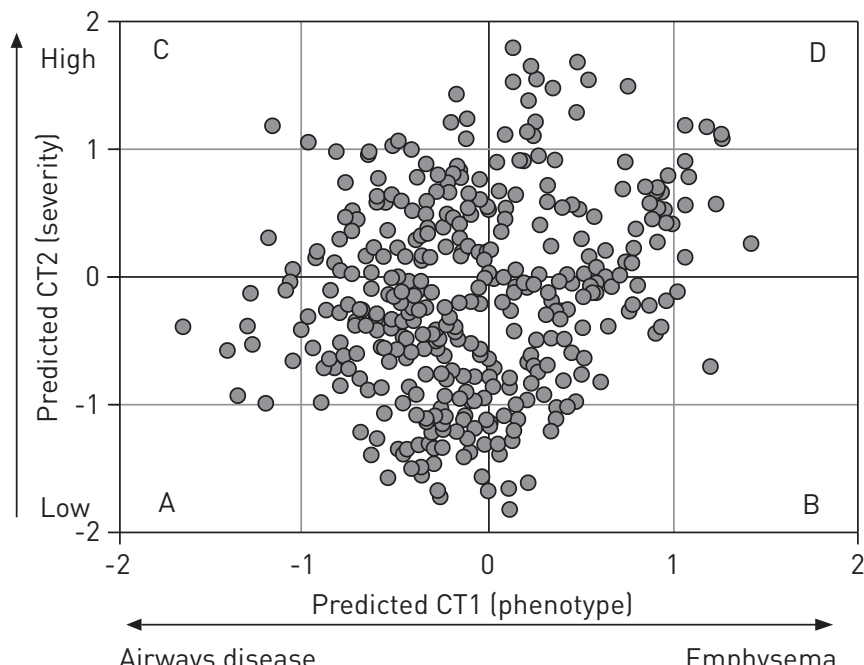

Airways disease
Emphysema
FIGURE 2 The distribution of the 373 patients of the testing set, using as coordinates the values of indexes predicted by multivariate regression models (CT1 (phenotype) and CT2 (severity)) computed by linearly combining the coefficients of the models developed from the learning set with the clinical and functional data of each patient of the testing set. Patient distribution is partitioned into four subsets (A, B, C and $\mathrm{D})$ by two perpendicular lines drawn from the zero values of CT1 and CT2.

prevalence of severe and very severe mMRC dyspnoea scores was significantly higher $(\mathrm{p}<0.01)$ in the $\mathrm{B}$ and D subsets (see online supplementary table E1). The large number of patients (120 out of 373, 32\%) of the testing set whose location in the graph is close to the predicted CT1 zero line (between -0.25 and 0.25 CT1 values) may have intermediate mechanisms of airflow limitation. Irrespective of the pathogenetic mechanism (predominant conductive airway obstruction in $\mathrm{A}$ and $\mathrm{C}$ and parenchymal destruction in $\mathrm{B}$ and D), the severity of COPD progressively worsens with increasing values of predicted CT2.

\section{Discussion}

The main finding of this study is that pulmonary function and sputum characteristics can be used in multivariate models to classify COPD patients according to the overall severity of the disease and the prevailing mechanism of airflow limitation as assessed by quantitative CT analysis. Results have been obtained using a data-driven statistical procedure. The models have been derived from a series of patients undergoing clinical, pulmonary function and quantitative CT scan evaluation and have been cross-validated and prospectively applied to classify in a bidimensional diagram a different larger series of patients undergoing only clinical and functional evaluation. The coordinates of the diagram (predicted CT1 (phenotype) and predicted CT2 (severity)) can be estimated by measuring FEV1/VC, TLC, FRC and DLCO and by determining whether the patient has chronic purulent sputum. CT parenchymal destruction and conductive airway thickening progressively prevail as the main mechanism of airflow limitation with positive or negative values of CT1, respectively. CT severity of the disease progressively increases with increasing values of CT2.

TABLE 6 Mean values of the pulmonary function tests in the four subsets of the 373 patients of the testing set subdivided according to the values of predicted computed tomography (CT) indexes CT1 (phenotype) and CT2 (severity)

Subset

\begin{tabular}{|c|c|c|c|c|}
\hline & \\
\hline & A & B & C & D \\
\hline Subjects $\mathrm{n}$ & 143 & 77 & 80 & 73 \\
\hline Predicted $\mathrm{CT}$ index range & $\mathrm{CT} 1<0$ СТ $2<0$ & $\mathrm{CT} 1>0 \mathrm{CT} 2<0$ & $\mathrm{CT} 1<0$ CT $2>0$ & $\mathrm{CT} 1>0 \mathrm{CT} 2>0$ \\
\hline $\mathrm{FEV}_{1} / \mathrm{VC}$ & 60 & 52 & 45 & 36 \\
\hline TLC $\%$ & 97 & 103 & 110 & 123 \\
\hline RV \% & 112 & 128 & 150 & 189 \\
\hline
\end{tabular}

All values are significantly different $(p<0.05)$ among the four subsets of patients. A, B, C, D: Subsets of patients subdivided according to the values of predicted computed tomography indexes (CT1 and CT2) as shown in figure 2. FEV1: forced expiratory volume in 1 s; VC: vital capacity; TLC: total lung capacity; RV: residual volume; FRC: functional residual capacity; ; DLCO: single breath diffusion capacity of the lung for carbon monoxide. 
DLCO is the functional variable accounting for the greatest variance in estimating CT1. This indicates that parenchymal destruction as assessed anatomically by reduced CT lung density and functionally by reduced diffusing capacity is an important determinant of the clinical phenotype of COPD. FEV $1 / \mathrm{VC}$ is the functional variable accounting for the greatest variance in estimating CT2. This indicates that airflow limitation, as assessed anatomically by both reduced CT density and increased airway wall thickness and functionally by FEV1/VC, is an important determinant of COPD severity. FEV1/VC instead of FEV1/FVC is entered in the model predicting COPD severity. This indicates that the use of VC at the denominator of the ratio could increase its sensitivity and accuracy in defining the severity of expiratory airflow limitation. Functional indexes of hyperinflation (TLC and FRC) subsequently enter in both models and slightly reduce the variance left after the first step of the multivariate regression process. Among the clinical variables, only chronic purulent sputum enters in both models. However, the regression coefficient connected with sputum purulence is negative for CT1 (phenotype) and positive for CT2 (severity). This indicates that presence of chronic purulent sputum, besides being an important determinant of the COPD clinical phenotype, namely predominant chronic inflammatory obstruction of the conductive airways, is also a marker of increased COPD severity.

Hence, the position assumed in the bidimensional CT1-CT2 diagram classifies each patient in relation to the prevailing mechanism of airflow limitation and the severity of the disease. The wide distribution of patients obtained in the present study is in agreement with the notion that COPD can have a continuous and wide spectrum of presentations in terms of clinical phenotype and disease severity. The strong relationships of model-predicted CT2 with indexes of both airflow obstruction and hyperinflation measured in the testing set (table 5) indicates that this parameter does reflect the overall lung dysfunction that characterises COPD. Further convincing support for the use of CT2 as an indicator of COPD severity should be obtained by comparing this variable with other markers of disease severity such as 6-min walking test, frequency of exacerbations and long-term survival.

The complexity of COPD cannot reliably be reflected by simple spirometric determinations of FEV1 [1] or by adding symptoms scores and exacerbation frequency to FEV1, as has been suggested [10]. The approach presented here seems to have the advantage of predicting at the same time the predominant COPD phenotype (conductive airway obstruction with negative CT1 values, intermediate with CT1 values close to zero and parenchymal destruction with positive CT1 values) and the degree of severity (ranging from mild to very severe with CT2 values progressively increasing from negative to positive). Prediction of CT1 and CT2 can be obtained objectively by the numerical values of pulmonary function evaluation and by the answer to the question about presence or absence of chronic purulent sputum. The resulting information could possibly be used to identify disease subgroups that may have different responses to treatment, and this may accelerate progress toward the development of specifically targeted therapeutic strategies, as currently recommended $[18,35]$.

Numerous studies have related clinical and full pulmonary function testing with quantitative CT in COPD $[2,11-14,18,22-27,36]$. Published relationships, however, are not readily comparable with the results obtained in this study, since, to the best of our knowledge, only the present report combines, via a datadriven statistical procedure, quantitative CT data with overlapping relationships, such as parenchymal destruction and conductive airway thickening.

The main limitation of this paper is that the series of patients from which CT1 (phenotype) and CT2 (severity) have been derived (learning set) is relatively small to be considered representative of the whole spectrum of COPD lung structural changes and, in this context, it is probably necessary to derive predictive multivariate models from larger samples of patients which could represent more accurately the general population of COPD. However, the multivariate models derived from the present learning set were able to prospectively classify a larger testing set differing in age and COPD severity. Another limitation of the paper is that the enrolment of patients on the basis of a post-bronchodilator $\mathrm{FEV} 1 / \mathrm{FVC}<70 \%$ according to GOLD guidelines [1], instead of using the lower limit of normality as suggested by the ATS/ERS guidelines [30], could have introduced a selection bias leading to overdiagnosis of COPD in male elderly nonsmokers and underdiagnosis in young smokers of both sexes [37].

Definite strengths of this work are related to the acquisition and analysis of functional and CT scan data. In fact, all data were obtained by specifically trained personnel working under standardised acquisition rules with the same pulmonary function and CT equipment and were analysed by an unbiased statistical procedure.

In summary, the data presented provide evidence that lung structural changes measured by CT scan are associated with clinical and lung function changes that may be used for an objective classification of the predominant phenotype and the severity of COPD. If these or similar multivariate models are confirmed in 
larger population studies, each COPD patient, despite being the individual expression of a wide and continuous spectrum of clinical presentations, after full pulmonary function testing and a short question about sputum characteristics, could be located on a diagram whose coordinates may indicate the type and severity of the structural changes underlying the mechanism of expiratory airflow limitation.

\section{References}

Rabe KF, Hurd S, Anzueto A, et al. Global strategy for the diagnosis, management, and prevention of chronic obstructive pulmonary disease: GOLD executive summary. Am J Respir Crit Care Med 2007; 176: 532-555.

2 Gelb AF, Hogg JC, Müller NL, et al. Contribution of emphysema and small airways in COPD. Chest 1996; 109: 353-359.

Wedzicha JA. The heterogeneity of chronic obstructive pulmonary disease. Thorax 2000; 55: 631-632.

4 Friedlander AL, Lynch D, Dyar LA, et al. Phenotypes of chronic obstructive pulmonary disease. COPD 2007; 4: 355-384.

Reilly JJ. COPD and declining FEV1 - time to divide and conquer? N Engl J Med 2008; 359: 1616-1618.

Pistolesi M. Beyond airflow limitation: another look at COPD. Thorax 2009; 64: $2-4$.

Burrows B, Fletcher CM, Heard BE, et al. The emphysematous and bronchial types of chronic airways obstruction. A clinicopathological study of patients in London and Chicago. Lancet 1966; 1: 830-835.

8 Han MK, Agusti A, Calverley PM, et al. Chronic obstructive pulmonary disease phenotypes: the future of COPD. Am J Respir Crit Care Med 2010; 182: 598-604.

9 Hurst JR, Vestbo J, Anzueto A, et al. Susceptibility to exacerbation in chronic obstructive pulmonary disease. N Engl J Med 2010; 363: 1128-1138.

10 Global Initiative for Chronic Obstructive Lung Disesae. Global Strategy for Diagnosis, Management, and Prevention of COPD, 2011. www.goldcopd.org/guidelines-global-strategy-for-diagnosis-management.html Date last accessed: August 8, 2012. Date last updated: February 2013.

11 Nakano Y, Muro S, Sakai H, et al. Computed tomographic measurements of airway dimensions and emphysema in smokers. Correlation with lung function. Am J Respir Crit Care Med 2000; 162: 1102-1108.

12 Camiciottoli G, Bartolucci M, Maluccio NM, et al. Spirometrically gated high-resolution CT findings in COPD: lung attenuation $v s$ lung function and dyspnea severity. Chest 2006; 129: 558-564.

13 Aziz ZA, Wells AU, Desai SR, et al. Functional impairment in emphysema: contribution of airway abnormalities and distribution of parenchymal disease. AJR Am J Roentgenol 2005; 185: 1509-1515.

14 Orlandi I, Moroni C, Camiciottoli G, et al. Chronic obstructive pulmonary disease: thin-section CT measurement of airway wall thickness and lung attenuation. Radiology 2005; 234: 604-610.

15 Pistolesi M, Camiciottoli G, Paoletti M, et al. Identification of a predominant COPD phenotype in clinical practice. Respir Med 2008; 102: 367-376.

16 Weatherall M, Travers J, Shirtcliffe PM, et al. Distinct clinical phenotypes of airways disease defined by cluster analysis. Eur Respir J 2009; 34: 812-818.

17 Garcia-Aymerich J, Gómez FP, Benet M, et al. Identification and prospective validation of clinically relevant chronic obstructive pulmonary disease (COPD) subtypes. Thorax 2011; 66: 430-437.

18 Han MK, Kazerooni EA, Lynch DA, et al. Chronic obstructive pulmonary disease exacerbations in the COPDGene study: associated radiologic phenotypes. Radiology 2011; 261: 274-282.

19 Nakano Y, Müller NL, King GG, et al. Quantitative assessment of airway remodelling using high-resolution CT. Chest 2002; 122: Suppl. 6, 271S-275S.

20 Hasegawa M, Nasuhara Y, Onodera Y, et al. Airflow limitation and airway dimensions in chronic obstructive pulmonary disease. Am J Respir Crit Care Med 2006; 173: 1309-1315.

21 Kim V, Han MK, Vance GB, et al. The chronic bronchitic phenotype of COPD: an analysis of the COPDGene study. Chest 2011; 140: 626-633.

22 Patel BD, Coxson HO, Pillai SG, et al. Airway wall thickening and emphysema show independent familial aggregation in chronic obstructive pulmonary disease. Am J Respir Crit Care Med 2008; 178: 500-505.

23 Han MK, Bartholmai B, Liu LX, et al. Clinical significance of radiologic characterizations in COPD. COPD 2009; 6 : 459-467.

24 Grydeland TB, Dirksen A, Coxson HO, et al. Quantitative computed tomography measures of emphysema and airway wall thickness are related to respiratory symptoms. Am J Respir Crit Care Med 2010; 181: 353-359.

25 Diaz AA, Valim C, Yamashiro T, et al. Airway count and emphysema assessed by chest CT imaging predicts clinical outcome in smokers. Chest 2010; 138: 880-887.

26 Martinez $\mathrm{CH}$, Chen $\mathrm{YH}$, Westgate PM, et al. Relationship between quantitative CT metrics and health status and BODE in chronic obstructive pulmonary disease. Thorax 2012; 67: 399-406.

27 Camiciottoli G, Bigazzi F, Bartolucci M, et al. Bode-index, modified Bode-index and ADO-score in chronic obstructive pulmonary disease: relationship with COPD phenotypes and CT lung density changes. COPD 2012; 9: 297-304.

28 Sarma A, Heilbrun ME, Conner KE, et al. Radiation and chest CT examinations: what do we know? Chest 2012; 142: 750-760.

29 Grydeland TB, Dirksen A, Coxson HO, et al. Quantitative computed tomography: emphysema and airway wall thickness by sex, age and smoking. Eur Respir J 2009; 34: 858-865.

30 Pellegrino R, Viegi G, Brusasco V, et al. Interpretative strategies for lung function tests. Eur Respir J 2005; 26: 948-968.

31 Hoffman EA, Simon BA, McLennan G. State of the art. A structural and functional assessment of the lung via multidetector-row computed tomography: phenotyping chronic obstructive pulmonary disease. Proc Am Thorac Soc 2006; 3: 519-532.

32 Zach JA, Newell JD Jr, Schroeder J, et al. Quantitative computed tomography of the lungs and airways in health nonsmoking adults. Invest Radiol 2012; 47: 596-602.

33 Larose DT. Data Mining Methods and Models. New York, John Wiley \& Sons, 2006. 
Kohavi R. A study of cross-validation and bootstrap for accuracy estimation and model selection. Proceedings of the Fourteenth International Joint Conference on Artificial Intelligence. San Mateo, Morgan Kaufmann, 1995. Fingleton J, Weatherall M, Beasley R. Towards individualised treatment in COPD. Thorax 2011; 66: 363-364.

36 Hesselbacher SE, Ross R, Schabath MB, et al. Cross-sectional analysis of the utility of pulmonary function tests in predicting emphysema in ever-smokers. Int J Environ Res Public Health 2011; 8: 1324-1340.

37 Brusasco V. Spirometric definition of COPD: exercise in futility or factual debate? Thorax 2012; 67: 569-570. 